\title{
Existence and Controllability Results for Fractional Impulsive Integrodifferential Systems in Banach Spaces
}

\author{
Haiyong Qin, Xin Zuo, and Jianwei Liu \\ Department of Automation, China University of Petroleum, Beijing 102249, China \\ Correspondence should be addressed to Jianwei Liu; liujw@cup.edu.cn
}

Received 4 March 2013; Revised 28 April 2013; Accepted 30 April 2013

Academic Editor: Rodrigo Lopez Pouso

Copyright ( 2013 Haiyong Qin et al. This is an open access article distributed under the Creative Commons Attribution License, which permits unrestricted use, distribution, and reproduction in any medium, provided the original work is properly cited.

\begin{abstract}
We firstly study the existence of PC-mild solutions for impulsive fractional semilinear integrodifferential equations and then present controllability results for fractional impulsive integrodifferential systems in Banach spaces. The method we adopt is based on fixed point theorem, semigroup theory, and generalized Bellman inequality. The results obtained in this paper improve and extend some known results. At last, an example is presented to demonstrate the applications of our main results.
\end{abstract}

\section{Introduction}

Fractional calculus is an area having a long history whose infancy dates back to three hundred years. However, at the beginning of fractional calculus, it develops slowly due to the disadvantage of technology. In recent decades, as the ancient mathematicians expected, fractional differential equations have been found to be a powerful tool in many fields, such as viscoelasticity, electrochemistry, control, porous media, and electromagnetic. For basic facts about fractional derivative and fractional calculus, one can refer to the books [1-4]. Since the fractional theory has played a very significant role in engineering, science, economy, and many other fields, during the past decades, fractional differential equations have attracted many authors, and there has been a great deal of interest in the solutions of fractional differential equations in analytical and numerical sense (see, e.g., [5-10] and references therein).

On the other hand, the impulsive differential systems are used to describe processes which are subjected to abrupt changes at certain moments [11-13]. The study of dynamical systems with impulsive effects has been an object of intensive investigations. It is well known that controllability is a key topic for control theory. Controllability means that it is possible to steer any initial state of the system to any final state in some finite time using an admissible control. We refer the readers to the survey [14] and the reference therein for controllability of nonlinear systems in Banach spaces. The sufficient controllability conditions for fractional impulsive integrodifferential systems in Banach spaces have already been obtained in [15-18].

Balachandran and Park [17] studied the controllability of fractional integrodifferential systems in Banach spaces without impulse

$$
\begin{gathered}
\frac{d^{q} x(t)}{d t^{q}}=A x(t)+f\left(t, x(t), \int_{0}^{t} h(t, s, x(s)) d s\right) \\
+B u(t), \quad t \in J=[0, b], \\
x(0)=x_{0} \in \mathbb{X}
\end{gathered}
$$

where $0<q<1$, the state $x(\cdot)$ takes values in the Banach space $\mathbb{X}, f: J \times \mathbb{X} \times \mathbb{X} \rightarrow \mathbb{X}, h: \Delta \times \mathbb{X} \rightarrow \mathbb{X}$ are continuous functions, and here $\Delta=\{(t, s): 0 \leq s \leq t \leq b\}$. The control function $u \in L^{2}[J, U]$, a Banach space of admissible control functions with $U$ as a Banach space, and $B: U \rightarrow \mathbb{X}$ is a bounded linear operator.

In [19], Mophou considered the existence and uniqueness of a mild solution for impulsive fractional semilinear differential equation

$$
\begin{gathered}
D_{t}^{\alpha} x(t)=A x(t)+f(t, x(t)), \quad t \in I=[0, T], t \neq t_{k}, \\
x(0)=x_{0} \in \mathbb{X}, \\
\left.\Delta x\right|_{t=t_{k}}=I_{k}\left(x\left(t_{k}^{-}\right)\right), \quad k=1,2, \ldots, m,
\end{gathered}
$$


where $D_{t}^{\alpha}$ is the Caputo fractional derivative, and $0<\alpha<1$. The operator $A: D(A) \subset \mathbb{X} \rightarrow \mathbb{X}$ is a generator of $\mathscr{C}_{0^{-}}$ semigroup $(T(t))_{t \geq 0}$ on a Banach space $\mathbb{X}$, and $I_{k}: \mathbb{X} \rightarrow \mathbb{X}$ are impulsive functions.

To consider fractional systems in the infinite dimensional space, the first important step is to define a new concept of the mild solution. Unfortunately, By Hernández et al. [20], we know that the concept of mild solutions used in [15-17, 19], inspired by Jaradat et al. [21], was not suitable for fractional evolution systems at all. Therefore, it is necessary to restudy this interesting and hot topic again.

Recently, in Wang and Zhou [18], a suitable concept of mild solutions was introduced, using Krasnoselskii's fixed point theorem and Sadovskii's fixed point theorem, investigating complete controllability of fractional evolution systems in the infinite dimensional spaces

$$
\begin{gathered}
{ }^{c} D_{t}^{q} x(t)=A x(t)+f(t, x(t))+B u(t), \\
t \in J=[0, b], \\
x(0)=x_{0} \in \mathbb{X},
\end{gathered}
$$

where ${ }^{c} D_{t}^{q}$ is the Caputo fractional derivative of the order $0<q \leq 1$ with the lower limit zero, the state $x(\cdot)$ takes values in Banach space $\mathbb{X}$, and the control function $u(\cdot)$ is given in $L^{2}[J, U]$, with $U$ as a Banach space. $A: D(A) \subset \mathbb{X} \rightarrow \mathbb{X}$ is the infinitesimal generator of a strongly continuous semigroup $(T(t))_{t \geq 0}$ in $\mathbb{X}, B$ is a bounded linear operator from $U$ to $\mathbb{X}$, and $f: J \times \mathbb{X} \rightarrow \mathbb{X}$ is given $\mathbb{X}$-value functions. Some sufficient conditions for complete controllability of the previous system were obtained.

Inspired by the work of the previous papers and many known results in [22-24], we study the existence of mild solutions for impulsive fractional semilinear integrodifferential equation

$$
\begin{array}{r}
D_{t}^{q} x(t)=A x(t)+f(t, x(t),(H x)(t)), \\
t \in I=[0, b], t \neq t_{k}, \\
x(0)=x_{0} \in \mathbb{X}, \\
\left.\Delta x\right|_{t=t_{k}}=I_{k}\left(x\left(t_{k}^{-}\right)\right), \quad k=1,2, \ldots, m,
\end{array}
$$

where $D_{t}^{q}$ is the Caputo fractional derivative, $0<q<1$, the state $x(\cdot)$ takes values in Banach space $\mathbb{X} . A: D(A) \subset \mathbb{X} \rightarrow$ $\mathbb{X}$ is the infinitesimal generator of a strongly continuous semigroup $(T(t))_{t \geq 0}$ of a uniformly bounded operator on $\mathbb{X}$, and $A$ is a bounded linear operator. $f: J \times \mathbb{X} \times \mathbb{X} \rightarrow \mathbb{X}$ is given $\mathbb{X}$-value functions, $H$ is defined as

$$
(H x)(t)=\int_{0}^{t} h(t, s, x(s)) d s,
$$

where $h: \Delta \times \mathbb{X} \rightarrow \mathbb{X}$ are continuous, here $\Delta=\{(t, s): 0 \leq$ $s \leq t \leq b\}, I_{k}: \mathbb{X} \rightarrow \mathbb{X}$ are impulsive functions, $0=t_{0}<$ $t_{1}<\cdots<t_{m}<t_{m+1}=b,\left.\Delta x\right|_{t=t_{k}}=x\left(t_{k}^{+}\right)-x\left(t_{k}^{-}\right)$, and $x\left(t_{k}^{+}\right)=$ $\lim _{h \rightarrow 0^{+}} x\left(t_{k}+h\right)$ and $x\left(t_{k}^{-}\right)=\lim _{h \rightarrow 0^{-}} x\left(t_{k}+h\right)$ represent the right and left limits of $x(t)$ at $t=t_{k}$, respectively.

We also define a control $u$ and present controllability results for fractional integrodifferential systems in Banach spaces

$$
\begin{array}{r}
D_{t}^{q} x(t)=A x(t)+f(t, x(t),(H x)(t))+B u(t), \\
t \in I=[0, b], t \neq t_{k}, \\
x(0)=x_{0} \in \mathbb{X}, \\
\left.\Delta x\right|_{t=t_{k}}=I_{k}\left(x\left(t_{k}^{-}\right)\right), \quad k=1,2, \ldots, m,
\end{array}
$$

where $B$ is a bounded linear operator from $U$ to $\mathbb{X}$, and the control function $u(\cdot)$ is given in $L^{2}[J, U]$, with $U$ as a Banach space. The method we adopt is based on the ideas in [1719, 22-24]. Compared with the previous results, this paper has three advantages. Firstly, we add operator $H$ in the nonlinear term $f$ and introduce a suitable concept of mild solutions of (4) and (6). Secondly, we not only study the existence of PC-mild solutions for impulsive fractional semilinear integrodifferential equation (4) but also present controllability results for fractional impulsive integrodifferential systems (6), and the results in $[17,19]$ could be seen as the special cases. Thirdly, our method avoids the compactness conditions on the semigroup $(T(t))_{t \geq 0}$, and some other hypotheses are more general compared with the previous research (see the conditions $\left(H_{1}\right)-\left(H_{3}\right)$ and $\left.\left(H_{5}\right)-\left(H_{8}\right)\right)$.

The rest of the paper is organized as follows. In Section 2, we present some preliminaries and lemmas that are to be used later to prove our main results. In Section 3, the existence of PC-mild solutions for (4) is discussed. In Section 4, by introducing a class of controls, we present the controllability results for fractional impulsive integrodifferential systems (6). In Section 5, an example is given to illustrate the theory.

\section{Preliminaries and Lemmas}

Let us consider the set of functions $\mathrm{PC}[I, \mathbb{X}]=\{x: I \rightarrow$ $\mathbb{X}: x \in C\left[\left(t_{k}, t_{k+1}\right), \mathbb{X}\right]$, and there exist $x\left(t_{k}^{-}\right)$and $x\left(t_{k}^{+}\right)$, $k=0,1,2, \ldots, m$ with $\left.x\left(t_{k}^{-}\right)=x\left(t_{k}\right)\right\}$. Endowed with the norm $\|x\|_{\mathrm{PC}}=\sup _{t \in I}\|x(t)\|$, it is easy to know that $(\mathrm{PC}[I, \mathbb{X}]$, $\left.\|\cdot\|_{\mathrm{PC}}\right)$ is a Banach space. Throughout this paper, let $A$ be the infinitesimal generator of a $C_{0}$-semigroup $(T(t))_{t \geq 0}$ of a uniformly bounded operators on $\mathbb{X}$. Let $L_{B}(\mathbb{X})$ be the Banach space of all linear and bounded operator on $\mathbb{X}$. For a $C_{0}$ semigroup $(T(t))_{t \geq 0}$, we set $M_{1}=\sup _{t \in I}\|T(t)\|_{L_{B}(\mathbb{X})}$. For each positive constant $r$, set $B_{r}=\{x \in \mathrm{PC}[I, \mathbb{X}]:\|x\| \leq r\}$.

Definition 1. The fractional integral of order $\gamma$ with the lower limit zero for a function $f$ is defined as

$$
I^{\gamma} f(t)=\frac{1}{\Gamma(\gamma)} \int_{0}^{t} \frac{f(s)}{(t-s)^{1-\gamma}} d s, \quad t>0, \gamma>0,
$$

provided that the right side is point-wise defined on $[0,+\infty)$, where $\Gamma(\cdot)$ is the gamma function. 
Definition 2. The Riemann-Liouville derivative of the order $\gamma$ with the lower limit zero for a function $f:[0, \infty] \rightarrow R$ can be written as

$$
\begin{aligned}
{ }^{L} D^{\gamma} f(t)= & \frac{1}{\Gamma(n-\gamma)} \frac{d^{n}}{d t^{n}} \\
& \times \int_{0}^{t} \frac{f(s)}{(t-s)^{1-n+\gamma}} d s, \quad t>0, n-1<\gamma<n .
\end{aligned}
$$

Definition 3. The Caputo derivative of the order $\gamma$ for a function $f:[0, \infty] \rightarrow R$ can be written as

$$
\begin{array}{r}
D^{\gamma} f(t)={ }^{L} D^{\gamma}\left(f(t)-\sum_{k=0}^{n-1} \frac{t^{k}}{k !} f^{(k)}(0)\right), \\
t>0, n-1<\gamma<n .
\end{array}
$$

Remark 4. (1) If $f(t) \in C^{n}[0, \infty)$, then

$$
\begin{aligned}
D^{\gamma} f(t) & =\frac{1}{\Gamma(n-\gamma)} \int_{0}^{t} \frac{f^{(n)}(s)}{(t-s)^{1-n+\gamma}} d s \\
& =I^{n-r} f^{(n)}(t), \quad t>0, n-1<\gamma<n .
\end{aligned}
$$

(2) The Caputo derivative of a constant is equal to zero.

(3) If $f$ is an abstract function with values in $\mathbb{X}$, then integrals which appear in Definitions 1, 2, and 3 are taken in Bochner's sense.

Definition 5 (see [22]). A mild solution of the following nonhomogeneous impulsive linear fractional equation of the form

$$
\begin{gathered}
D_{t}^{q} x(t)=A x(t)+h(t), \quad t \in I=[0, b], 0<q<1, t \neq t_{k}, \\
x(0)=x_{0} \in \mathbb{X}, \\
\left.\Delta x\right|_{t=t_{k}}=I_{k}\left(x\left(t_{k}^{-}\right)\right), \quad k=1,2, \ldots, m,
\end{gathered}
$$

is given by

$$
x(t)=\left\{\begin{array}{cc}
\mathscr{T}(t) x_{0}+\int_{0}^{t}(t-s)^{q-1} \mathcal{S}(t-s) h(s) d s, & t \in\left[0, t_{1}\right], \\
\mathscr{T}(t) x_{0}+\mathscr{T}\left(t-t_{1}\right) I_{1}\left(x\left(t_{1}^{-}\right)\right) & \\
+\int_{0}^{t}(t-s)^{q-1} \mathcal{S}(t-s) h(s) d s, & t \in\left(t_{1}, t_{2}\right], \\
\vdots & \\
\mathscr{T}(t) x_{0}+\sum_{k=1}^{m} \mathscr{T}\left(t-t_{k}\right) I_{k}\left(x\left(t_{k}^{-}\right)\right) & \\
+\int_{0}^{t}(t-s)^{q-1} \mathcal{S}(t-s) h(s) d s, & t \in\left(t_{m}, b\right],
\end{array}\right.
$$

where $\mathscr{T}(\cdot)$ and $\mathcal{S}(\cdot)$ are called characteristic solution operators and given by

$$
\begin{gathered}
\mathscr{T}(t)=\int_{0}^{\infty} \xi_{q}(\theta) T\left(t^{q} \theta\right) d \theta, \\
\mathcal{S}(t)=q \int_{0}^{\infty} \theta \xi_{q}(\theta) T\left(t^{q} \theta\right) d \theta,
\end{gathered}
$$

and for $\theta \in(0, \infty)$,

$$
\begin{gathered}
\xi_{q}(\theta)=\frac{1}{q} \theta^{-1-(1 / q)} \omega_{q}\left(\theta^{-1 / q}\right) \geq 0, \\
\omega_{q}(\theta)=\frac{1}{\pi} \sum_{n=1}^{\infty}(-1)^{n-1} \theta^{-q n-1} \frac{\Gamma(n q+1)}{n !} \sin (n \pi q),
\end{gathered}
$$

where $\xi_{q}$ is a probability density function defined on $(0, \infty)$; that is,

$$
\xi_{q}(\theta) \geq 0, \theta \in(0, \infty), \quad \int_{0}^{\infty} \xi_{q}(\theta) d \theta=1 .
$$

Definition 6. By a PC-mild solution of (4), we mean that a function $x \in \mathrm{PC}[I, \mathbb{X}]$, which satisfies the following integral equation:

$$
x(t)=\left\{\begin{array}{c}
\mathscr{T}(t) x_{0}+\int_{0}^{t}(t-s)^{q-1} \mathcal{S}(t-s) \\
\quad \times f(s, x(s),(H x)(s)) d s, \quad t \in\left[0, t_{1}\right], \\
\mathscr{T}(t) x_{0}+\mathscr{T}\left(t-t_{1}\right) I_{1}\left(x\left(t_{1}^{-}\right)\right) \\
+\int_{0}^{t}(t-s)^{q-1} \mathcal{S}(t-s) \\
\times f(s, x(s),(H x)(s)) d s, \quad t \in\left(t_{1}, t_{2}\right], \\
\vdots \\
\mathscr{T}(t) x_{0}+\sum_{k=1}^{m} \mathscr{T}\left(t-t_{k}\right) I_{k}\left(x\left(t_{k}^{-}\right)\right) \\
+\int_{0}^{t}(t-s)^{q-1} \mathcal{S}(t-s)
\end{array}\right.
$$

Definition 7. By a PC-mild solution of the system (6), we mean that a function $x \in \mathrm{PC}[I, \mathbb{X}]$, which satisfies the following integral equation:

$$
x(t)=\left\{\begin{array}{c}
\mathscr{T}(t) x_{0}+\int_{0}^{t}(t-s)^{q-1} \mathcal{S}(t-s) \\
\times[f(s, x(s),(H x)(s)) \\
+B u(s)] d s, \\
\mathscr{T}(t) x_{0}+\mathscr{T}\left(t-t_{1}\right) I_{1}\left(x\left(t_{1}^{-}\right)\right) \\
+\int_{0}^{t}(t-s)^{q-1} \mathcal{S}(t-s) \\
\times[f(s, x(s),(H x)(s)) \\
\left.+B u(s)] d s, t_{1}\right], \\
\vdots \\
\mathscr{T}(t) x_{0}+\sum_{k=1}^{m} \mathscr{T}\left(t-t_{k}\right) I_{k}\left(x\left(t_{k}^{-}\right)\right) \\
+\int_{0}^{t}(t-s)^{q-1} \mathcal{S}(t-s) \\
\times[f(s, x(s),(H x)(s)) \\
+B u(s)] d s,
\end{array}\right.
$$

Definition 8. The system (6) is said to be controllable on the interval $J$ if, for every $x_{0}, x_{1} \in \mathbb{X}$, there exists a control $u \in$ $L^{2}(J, U)$ such that a mild solution $x$ of (6) satisfies $x(b)=x_{1}$. 
Definition 9 (see [25]). Let $\mathbb{X}$ be a Banach space, and a one parameter family $T(t), 0 \leq t<+\infty$, of bounded linear operators from $\mathbb{X}$ to $\mathbb{X}$ is a semigroup of bounded linear operators on $\mathbb{X}$ if

(1) $T(0)=I$ (here, $I$ is the identity operator on $\mathbb{X}$ );

(2) $T(t+s)=T(t) T(s)$ for every $t, s \geq 0$ (the semigroup property).

A semigroup of bounded linear operator, $T(t)$, is uniformly continuous if $\lim _{t \downarrow 0}\|T(t)-I\|=0$.

Lemma 10 (see [25]). Linear operator $A$ is the infinitesimal generator of a uniformly continuous semigroup if and only if $A$ is a bounded linear operator.

Lemma 11 (see [19]). Let $T$ be a continuous and compact mapping of a Banach space $\mathbb{X}$ into itself, such that

$$
\{x \in \mathbb{X}: x=\lambda T x \text { for some } 0 \leq \lambda \leq 1\}
$$

is bounded. Then, T has a fixed point.

Lemma 12. The operators $\mathscr{T}(t)$ and $\mathcal{S}(t)$ have the following properties.

(i) For any fixed $t \geq 0, \mathscr{T}(t)$ and $\mathcal{S}(t)$ are linear and bounded operators; that is, for any $x \in \mathbb{X}$,

$\|\mathscr{T}(t) x\| \leq M_{1}\|x\|, \quad\|\mathcal{S}(t) x\| \leq \frac{q M_{1}}{\Gamma(1+q)}\|x\|$.

(ii) $\{\mathscr{T}(t), t \geq 0\}$ and $\{\delta(t), t \geq 0\}$ are strongly continuous.

(iii) $\{\mathscr{T}(t), t \geq 0\}$ and $\{\delta(t), t \geq 0\}$ are uniformly continuous; that is, for each fixed $t>0$, and $\epsilon>0$, there exists $h>0$ such that

$$
\begin{array}{cc}
\|\mathscr{T}(t+\epsilon)-\mathscr{T}(t)\| \leq \varepsilon, & \text { for } t+\epsilon \geq 0,|\epsilon|<h, \\
\|\mathcal{S}(t+\epsilon)-\mathcal{S}(t)\| \leq \varepsilon, & \text { for } t+\epsilon \geq 0,|\epsilon|<h .
\end{array}
$$

Proof. For the proof of (i) and (ii), the reader can refer to [23, Lemma 2.9] and [24, Lemmas 3.2-3.5]. For each fixed $t>0$, and $h>\epsilon>0$, one can obtain

$$
\begin{aligned}
& \|\mathscr{T}(t+\epsilon)-\mathscr{T}(t)\| \\
& \quad \leq \int_{0}^{\infty} \xi_{q}(\theta)\left\|T\left((t+\epsilon)^{q} \theta\right)-T\left(t^{q} \theta\right)\right\| d \theta \\
& \quad \leq M_{1} \int_{0}^{\infty} \xi_{q}(\theta)\left\|T\left((t+\epsilon)^{q} \theta-t^{q} \theta\right)-I\right\| d \theta, \\
& \|\mathcal{S}(t+\epsilon)-\mathcal{S}(t)\| \\
& \quad \leq q M_{1} \int_{0}^{\infty} \theta \xi_{q}(\theta)\left\|T\left((t+\epsilon)^{q} \theta-t^{q} \theta\right)-I\right\| d \theta .
\end{aligned}
$$

Because $A$ is a bounded linear operator, from Lemma 10 and Definition 9, we know that $A$ is the infinitesimal generator of a uniformly continuous semigroup. Thus, by the properties of uniformly continuous semigroup $(T(t))_{t \geq 0}$, we get

$$
\begin{gathered}
\|\mathcal{T}(t+\epsilon)-\mathscr{T}(t)\| \leq \varepsilon, \\
\|\mathcal{S}(t+\epsilon)-\mathcal{S}(t)\| \leq \varepsilon ;
\end{gathered}
$$

that is, $\{\mathscr{T}(t), t \geq 0\}$ and $\{\mathcal{S}(t), t \geq 0\}$ are uniformly continuous.

We list here the hypotheses to be used later.

$\left(H_{1}\right) f: I \times \mathbb{X} \times \mathbb{X} \rightarrow \mathbb{X}$ is continuous and there exist functions $\mu_{1}, \mu_{2} \in L\left[I, \mathbb{R}^{+}\right]$such that

$$
\begin{array}{r}
\left\|f\left(t, x_{1}, y_{1}\right)-f\left(t, x_{2}, y_{2}\right)\right\| \\
\leq \mu_{1}(t)\left\|x_{1}-x_{2}\right\|+\mu_{2}(t)\left\|y_{1}-y_{2}\right\|, \\
x_{1}, x_{2}, y_{1}, y_{2} \in \mathbb{X} .
\end{array}
$$

$\left(H_{2}\right) h: \Delta \times \mathbb{X} \times \mathbb{X} \rightarrow \mathbb{X}$ is continuous and there exist function $\nu_{1} \in C\left[I, \mathbb{R}^{+}\right]$such that

$\left\|h\left(t, s, x_{1}\right)-h\left(t, s, x_{2}\right)\right\| \leq v_{1}(t)\left\|x_{1}-x_{2}\right\|, \quad x_{1}, x_{2} \in \mathbb{X}$.

$\left(H_{3}\right)$ There exist $\omega_{k} \in C\left[I, \mathbb{R}^{+}\right]$such that

$$
\begin{gathered}
\left\|I_{k}\left(x_{1}\right)-I_{k}\left(x_{2}\right)\right\| \leq \omega_{k}(t)\left\|x_{1}-x_{2}\right\|, \\
x_{1}, x_{2} \in \mathbb{X}, \quad k=1,2, \ldots, m .
\end{gathered}
$$

$\left(H_{4}\right)$ The function $\Omega_{m}(t): I \rightarrow \mathbb{R}^{+}$is defined by

$$
\begin{aligned}
\Omega_{m}(t)= & \frac{q M_{1}}{\Gamma(1+q)} \int_{0}^{t}(t-s)^{q-1}\left(\mu_{1}(s)+v_{1}^{0} b \mu_{2}(s)\right) d s \\
& +m \omega_{0} M_{1},
\end{aligned}
$$

where $v_{1}^{0}=\max \left\{v_{1}(t) \mid t \in I\right\}, \omega_{0}=\max \left\{\omega_{k}(t) t \in\right.$ $I, k=1,2, \ldots, m\}$, and $0<\Omega_{m}(t)<1, t \in I$.

$\left(H_{4}^{\prime}\right)$ The constants $\Omega_{u}$ and $\Omega_{m}^{\prime}(t): I \rightarrow \mathbb{R}^{+}$are defined by

$$
\begin{aligned}
\Omega_{u}= & \frac{q M_{1} K}{\Gamma(1+q)} \int_{0}^{b}(b-s)^{q-1}\left(\mu_{1}(s)+\nu_{1}^{0} b \mu_{2}(s)\right) d s \\
& +\omega_{0} m M_{1}, \\
\Omega_{m}^{\prime}(t)= & \frac{q M_{1}}{\Gamma(1+q)} \int_{0}^{t}(t-s)^{q-1}\left(\mu_{1}(s)+\nu_{1}^{0} b \mu_{2}(s)\right) d s \\
& +\frac{q M_{1} \Omega_{u}}{\Gamma(1+q)} \int_{0}^{t}(t-s)^{q-1} d s+\omega_{0} m M_{1},
\end{aligned}
$$




\section{Existence of Mild Solutions}

Theorem 13. If the hypotheses $\left(H_{1}\right)-\left(H_{4}\right)$ are satisfied, then the fractional impulsive integrodifferential equation (4) has a unique mild solution $x \in P C[I, \mathbb{X}]$.

Proof. Define an operator $\mathrm{Q}$ on $\mathrm{PC}[I, \mathbb{X}]$ by

$$
(Q x)(t)=\left\{\begin{array}{c}
\mathscr{T}(t) x_{0}+\int_{0}^{t}(t-s)^{q-1} \mathcal{S}(t-s) \\
\times f(s, x(s), \\
(H x)(s)) d s, \\
\mathscr{T}(t) x_{0}+\mathscr{T}\left(t-t_{1}\right) I_{1}\left(x\left(t_{1}^{-}\right)\right) \\
+\int_{0}^{t}(t-s)^{q-1} \mathcal{S}(t-s) \\
\times f(s, x(s),(H x)(s)) d s, \quad t \in\left(t_{1}, t_{2}\right], \\
\vdots \\
\mathcal{T}(t) x_{0}+\sum_{k=1}^{m} \mathscr{T}\left(t-t_{k}\right) I_{k}\left(x\left(t_{k}^{-}\right)\right) \\
+\int_{0}^{t}(t-s)^{q-1} \mathcal{S}(t-s) \\
\times f(s, x(s),(H x)(s)) d s, \quad t \in\left(t_{m}, b\right] .
\end{array}\right.
$$

We will show that $Q$ is well defined on $\operatorname{PC}[I, \mathbb{X}]$. For $0 \leq \tau<$ $t \leq t_{1}$, applying (28), we obtain

$$
\begin{aligned}
& \|(Q x)(t)-(Q x)(\tau)\| \\
& \leq\|\mathscr{T}(t)-\mathscr{T}(\tau)\|\left\|x_{0}\right\| \\
& +\| \int_{\tau}^{t}(t-s)^{q-1} \mathcal{S}(t-s) f(s, x(s),(H x)(s)) d s \\
& +\int_{0}^{\tau}(t-s)^{q-1} \mathcal{S}(t-s) f(s, x(s),(H x)(s)) d s \\
& +\int_{0}^{\tau}(t-s)^{q-1} \mathcal{S}(\tau-s) f(s, x(s),(H x)(s)) d s \\
& -\int_{0}^{\tau}(t-s)^{q-1} \mathcal{S}(\tau-s) f(s, x(s),(H x)(s)) d s \\
& -\int_{0}^{\tau}(\tau-s)^{q-1} \mathcal{S}(\tau-s) f(s, x(s),(H x)(s)) d s \| \\
& \leq\|\mathscr{T}(t)-\mathscr{T}(\tau)\|\left\|x_{0}\right\| \\
& +\left\|\int_{\tau}^{t}(t-s)^{q-1} \mathcal{S}(t-s) f(s, x(s),(H x)(s)) d s\right\| \\
& +\| \int_{0}^{\tau}(t-s)^{q-1}[\mathcal{S}(t-s)-\mathcal{S}(\tau-s)] \\
& \times f(s, x(s),(H x)(s)) d s \| \\
& +\| \int_{0}^{\tau}\left[(t-s)^{q-1}-(\tau-s)^{q-1}\right] \\
& \times \mathcal{S}(\tau-s) f(s, x(s),(H x)(s)) d s \| .
\end{aligned}
$$

From the well-known inequality $\left|t^{\sigma}-\tau^{\sigma}\right| \leq(t-\tau)^{\sigma}$ for $\sigma \in(0,1]$ and $0<\tau \leq t$ and Lemma 12 , it is obvious that $\|(Q x)(t)-(Q x)(\tau)\| \rightarrow 0$ as $t \rightarrow \tau$. Thus, we deduce that $Q x \in C\left[\left[0, t_{1}\right], \mathbb{X}\right]$.

For $t_{1}<\tau<t \leq t_{2}$, we have

$$
\begin{aligned}
& \|(Q x)(t)-(Q x)(\tau)\| \\
& \leq\|\mathscr{T}(t)-\mathscr{T}(\tau)\|\left\|x_{0}\right\| \\
& +\left\|\mathscr{T}\left(t-t_{1}\right)-\mathscr{T}\left(\tau-t_{1}\right)\right\|\left\|I_{1}\left(x\left(t_{1}^{-}\right)\right)\right\| \\
& +\left\|\int_{\tau}^{t}(t-s)^{q-1} \mathcal{S}(t-s) f(s, x(s),(H x)(s)) d s\right\| \\
& +\| \int_{0}^{\tau}(t-s)^{q-1}[\mathcal{S}(t-s)-\mathcal{S}(\tau-s)] \\
& \quad \times f(s, x(s),(H x)(s)) d s \| \\
& +\| \int_{0}^{\tau}\left[(t-s)^{q-1}-(\tau-s)^{q-1}\right] \\
& \quad \times \mathcal{S}(\tau-s) f(s, x(s),(H x)(s)) d s \| .
\end{aligned}
$$

It is easy to get that, as $t \rightarrow \tau$, the right-hand side of the previous inequality tends to zero. Thus, we can deduce that $Q x \in C\left[\left(t_{1}, t_{2}\right], \mathbb{X}\right]$. By repeating the same procedure, we can also obtain that $Q x \in C\left[\left(t_{2}, t_{3}\right], \mathbb{X}\right], \ldots, Q x \in C\left[\left(t_{m}, b\right], \mathbb{X}\right]$. That is, $Q x \in \mathrm{PC}[I, \mathbb{X}]$.

Take $t \in\left[0, t_{1}\right]$; then,

$$
\begin{gathered}
\|(Q x)(t)-(Q y)(t)\| \\
\leq \int_{0}^{t}(t-s)^{q-1} \| \mathcal{S}(t-s) \\
\times(f(s, x(s),(H x)(s)) \\
\quad-f(s, y(s),(H y)(s))) \| d s \\
\leq \frac{q M_{1}}{\Gamma(1+q)} \int_{0}^{t}(t-s)^{q-1} \\
\times\left(\mu_{1}(s)\|x(s)-y(s)\|\right. \\
\left.+\mu_{2}(s)\|(H x)(s)-(H y)(s)\|\right) d s .
\end{gathered}
$$

From $\left(\mathrm{H}_{2}\right)$ and $\left(\mathrm{H}_{4}\right)$, we obtain

$$
\begin{aligned}
& \|(Q x)(t)-(Q y)(t)\| \\
& \leq \frac{q M_{1}}{\Gamma(1+q)} \int_{0}^{t}(t-s)^{q-1}\left(\mu_{1}(s)+\nu_{1}^{0} b \mu_{2}(s)\right) \\
& \quad \times\|x(s)-y(s)\| d s .
\end{aligned}
$$

So we deduce that

$$
\begin{aligned}
& \|(Q x)(t)-(Q y)(t)\|_{\mathrm{PC}} \\
& \leq \frac{q M_{1}}{\Gamma(1+q)} \int_{0}^{t}(t-s)^{q-1}\left(\mu_{1}(s)+v_{1}^{0} b \mu_{2}(s)\right) d s \\
& \quad \times\|x-y\|_{\mathrm{PC}} .
\end{aligned}
$$


In general, for each $t \in\left(t_{i}, t_{i+1}\right], 1 \leq i \leq m$, using the assumptions,

$$
\begin{aligned}
& \|(Q x)(t)-(Q y)(t)\|_{\mathrm{PC}} \\
& \leq \frac{q M_{1}}{\Gamma(1+q)} \int_{0}^{t}(t-s)^{q-1}\left(\mu_{1}(s)+\nu_{1}^{0} b \mu_{2}(s)\right) d s\|x-y\|_{\mathrm{PC}} \\
& \quad+\| \sum_{k=1}^{i} \mathscr{T}\left(t-t_{k}\right) I_{k}\left(x\left(t_{k}^{-}\right)\right) \\
& \quad-\sum_{k=1}^{i} \mathscr{T}\left(t-t_{k}\right) I_{k}\left(y\left(t_{k}^{-}\right)\right) \| \\
& \leq\left(\frac{q M_{1}}{\Gamma(1+q)} \int_{0}^{t}(t-s)^{q-1}\left(\mu_{1}(t)+v_{1}^{0} b \mu_{2}(t)\right) d s\right. \\
& \left.\quad+i \omega_{0} M_{1}\right)\|x-y\|_{\mathrm{PC}} \\
& \leq \Omega_{i}(t)\|x-y\|_{\mathrm{PC}}
\end{aligned}
$$

when $i=m$, obviously

$$
\begin{aligned}
& \|(Q x)(t)-(Q y)(t)\|_{\mathrm{PC}} \\
& \leq\left(\frac{q M_{1}}{\Gamma(1+q)} \int_{0}^{t}(t-s)^{q-1}\left(\mu_{1}(s)+\nu_{1}^{0} b \mu_{2}(s)\right) d s\right. \\
& \left.\quad+m \omega_{0} M_{1}\right)\|x-y\|_{\mathrm{PC}} \\
& \leq \Omega_{m}(t)\|x-y\|_{\mathrm{PC} .}
\end{aligned}
$$

Noting that $\Omega_{i}(t) \leq \Omega_{m}(t)$, with assumption $\left(H_{4}\right)$ and in the view of the contraction mapping principle, we know that $Q$ has a unique fixed point $x \in \operatorname{PC}[I, \mathbb{X}]$; that is,

$$
x(t)=\left\{\begin{array}{c}
\mathscr{T}(t) x_{0}+\int_{0}^{t}(t-s)^{q-1} \mathcal{S}(t-s) \\
\quad \times f(s, x(s),(H x)(s)) d s, \quad t \in\left[0, t_{1}\right], \\
\mathscr{T}(t) x_{0}+\mathscr{T}\left(t-t_{1}\right) I_{1}\left(x\left(t_{1}^{-}\right)\right) \\
+\int_{0}^{t}(t-s)^{q-1} \mathcal{S}(t-s) \\
\times f(s, x(s),(H x)(s)) d s, \quad t \in\left(t_{1}, t_{2}\right], \\
\vdots \\
\mathscr{T}(t) x_{0}+\sum_{k=1}^{m} \mathscr{T}\left(t-t_{k}\right) I_{k}\left(x\left(t_{k}^{-}\right)\right) \\
+\int_{0}^{t}(t-s)^{q-1} \mathcal{S}(t-s)
\end{array}\right.
$$

is a PC-mild solution of (4).

In order to obtain results by the Schaefer fixed point theorem, let us list the following hypotheses.
$\left(H_{5}\right) f: I \times \mathbb{X} \times \mathbb{X} \rightarrow \mathbb{X}$ is continuous and there exist functions $\mu_{3}, \mu_{4}, \mu_{5} \in L\left[I, \mathbb{R}^{+}\right]$such that

$\|f(t, x, y)\| \leq \mu_{3}(t)+\mu_{4}(t)\|x\|+\mu_{5}(t)\|y\|, \quad t \in I, x, y \in \mathbb{X}$.

$\left(H_{6}\right) h: \Delta \times \mathbb{X} \times \mathbb{X} \rightarrow \mathbb{X}$ is continuous and there exist functions $v_{2}, v_{3} \in C\left[I, \mathbb{R}^{+}\right]$such that

$$
\|h(t, s, x)\| \leq v_{2}(s)+v_{3}(s)\|x\|, \quad x \in \mathbb{X}
$$

$\left(H_{7}\right)$ There exist $\psi_{k} \in C\left[I, \mathbb{R}^{+}\right]$such that

$$
\left\|I_{k}(x)\right\| \leq \psi_{k}(t)\|x\|, \quad x \in \mathbb{X} .
$$

$\left(H_{8}\right)$ For all bounded subsets $B_{r}$, the set

$$
\begin{aligned}
\Pi_{h, \delta}(t)=\left\{\mathscr{T}_{\delta}(t) x_{0}\right. \\
\quad+\int_{0}^{t-h}(t-s)^{q-1} \mathcal{S}_{\delta}(t-s) F(s) d s \\
\left.\quad+\sum_{k=1}^{m} \mathscr{T}_{\delta}\left(t-t_{k}\right) I_{k}\left(x\left(t_{k}^{-}\right)\right): x \in B_{r}\right\}
\end{aligned}
$$

is relatively compact in $\mathbb{X}$ for arbitrary $h \in(0, t)$ and $\delta>0$, where

$$
\begin{gathered}
\mathscr{T}_{\delta}(t)=\int_{\delta}^{\infty} \xi_{q}(\theta) T\left(t^{q} \theta\right) d \theta, \\
\mathcal{S}_{\delta}(t)=q \int_{\delta}^{\infty} \theta \xi_{q}(\theta) T\left(t^{q} \theta\right) d \theta .
\end{gathered}
$$

$\left(H_{8}^{\prime}\right)$ For all bounded subsets $B_{r}$, the set

$$
\begin{aligned}
& \Pi_{h, \delta}^{\prime}(t)=\left\{\mathscr{T}_{\delta}(t) x_{0}\right. \\
&+\int_{0}^{t-h}(t-s)^{q-1} \mathcal{\delta}_{\delta}(t-s)[F(s)+B u(s)] d s \\
&\left.+\sum_{k=1}^{m} \mathscr{T}_{\delta}\left(t-t_{k}\right) I_{k}\left(x\left(t_{k}^{-}\right)\right): x \in B_{r}\right\}
\end{aligned}
$$

is relatively compact in $\mathbb{X}$ for arbitrary $h \in(0, t)$ and $\delta>0$.

Theorem 14. If the hypotheses $\left(H_{5}\right)-\left(H_{8}\right)$ are satisfied, the fractional impulsive integrodifferential equation (4) has at least one mild solution $x \in P C[I, \mathbb{X}]$. 
Proof. From Theorem 13, we know that operator $Q$ is defined as follows:

$$
(Q x)(t)=\left\{\begin{array}{c}
\mathscr{T}(t) x_{0}+\int_{0}^{t}(t-s)^{q-1} \mathcal{S}(t-s) \\
\times f(s, x(s), \\
\mathscr{T}(t) x_{0}+\mathcal{T}\left(t-t_{1}\right) I_{1}\left(x\left(t_{1}^{-}\right)\right) \\
+\int_{0}^{t}(t-s)^{q-1} \mathcal{S}(t-s) \\
\times f(s, x(s),(H x)(s)) d s, \quad t \in\left(t_{1}, t_{2}\right], \\
\vdots \\
\mathscr{T}(t) x_{0}+\sum_{k=1}^{m} \mathscr{T}\left(t-t_{k}\right) I_{k}\left(x\left(t_{k}^{-}\right)\right) \\
+\int_{0}^{t}(t-s)^{q-1} \mathcal{S}(t-s) \\
\times f(s, x(s),(H x)(s)) d s, \quad t \in\left(t_{m}, b\right] .
\end{array}\right.
$$

We will prove the results in five steps.

Step 1 (continuity of $Q$ on $\left.\left(t_{i}, t_{i+1}\right](i=0,1,2, \ldots, m)\right)$. Let $x_{n}, x \in \mathrm{PC}[I, \mathbb{X}]$ such that $\left\|x_{n}-x^{*}\right\|_{\mathrm{PC}} \rightarrow 0(n \rightarrow+\infty)$, and then $r=\sup _{n}\left\|x_{n}\right\|_{\mathrm{PC}}<\infty$ and $\left\|x^{*}\right\|_{\mathrm{PC}}<r$; for every $t \in\left(t_{i}, t_{i+1}\right](i=0,1,2, \ldots, m)$, we have

$$
\begin{aligned}
& \left\|\left(Q x_{n}\right)(t)-(Q x)(t)\right\| \\
& \leq \frac{q M_{1}}{\Gamma(1+q)} \int_{0}^{t}(t-s)^{q-1} \\
& \quad \times \| f\left(s, x_{n}(s),\left(H x_{n}\right)(s)\right) \\
& \quad-f(s, x(s),(H x)(s)) \| d s \\
& +\left\|\sum_{k=1}^{m} \mathscr{T}\left(t-t_{k}\right) I_{k}\left(x_{n}\left(t_{k}^{-}\right)\right)-\sum_{k=1}^{m} \mathscr{T}\left(t-t_{k}\right) I_{k}\left(x\left(t_{k}^{-}\right)\right)\right\| .
\end{aligned}
$$

Since the functions $f$ and $I_{k}$ are continuous,

$$
\begin{gathered}
f\left(s, x_{n}(s),\left(H x_{n}\right)(s)\right) \longrightarrow f(s, x(s),(H x)(s)), \\
I_{k}\left(x_{n}\left(t_{k}^{-}\right)\right) \longrightarrow I_{k}\left(x\left(t_{k}^{-}\right)\right) \quad n \longrightarrow \infty .
\end{gathered}
$$

By conditions $\left(H_{5}\right)$ and $\left(H_{6}\right)$, we know that

$$
\begin{aligned}
&\left\|f\left(s, x_{n}(s),\left(H x_{n}\right)(s)\right)-f(s, x(s),(H x)(s))\right\| \\
& \leq 2 \mu_{3}(s)+\mu_{4}(s)\left(\|x\|+\left\|x_{n}\right\|\right) \\
&+\mu_{5}(s)\left(\|H x\|+\left\|H x_{n}\right\|\right) \\
& \leq 2 \mu_{3}(s)+2 \mu_{5}(s) \int_{0}^{s} v_{2}(\theta) d \theta \\
&+\left(\mu_{4}(s)+\mu_{5}(s) \int_{0}^{s} v_{3}(\theta) d \theta\right)\left(\|x\|+\left\|x_{n}\right\|\right)
\end{aligned}
$$

$$
\begin{aligned}
\leq & 2 \mu_{3}(s)+2 \mu_{5}(s) \int_{0}^{s} \nu_{2}(\theta) d \theta \\
& +\left(2 \mu_{4}(s)+2 \mu_{5}(s) \int_{0}^{s} \nu_{3}(\theta) d \theta\right) r .
\end{aligned}
$$

Hence,

$$
\begin{aligned}
(t-s)^{q-1} \| & f\left(s, x_{n}(s),\left(H x_{n}\right)(s)\right) \\
& -f(s, x(s),(H x)(s)) \| \in L^{1}\left[I, \mathbb{R}^{+}\right] .
\end{aligned}
$$

By the Lebesgue dominated convergence theorem, we get

$$
\begin{aligned}
\int_{0}^{t}(t-s)^{q-1} \| & f\left(s, x_{n}(s),\left(H x_{n}\right)(s)\right) \\
& -f(s, x(s),(H x)(s)) \| d s \longrightarrow 0 .
\end{aligned}
$$

It is easy to obtain that

$$
\lim _{n \rightarrow \infty}\left\|\left(Q x_{n}\right)(t)-(Q x)(t)\right\|_{P C}=0 .
$$

Thus, $Q$ is continuous on $\left(t_{i}, t_{i+1}\right](i=0,1,2, \ldots, m)$.

Step 2 (Q maps bounded sets into bounded sets in $\mathrm{PC}[I, \mathbb{X}]$ ). From (43), we get

$\|(Q x)(t)\|$

$$
\begin{aligned}
\leq & \left\|\mathscr{T}(t) x_{0}\right\|+\frac{q M_{1}}{\Gamma(1+q)} \\
& \times \int_{0}^{t}(t-s)^{q-1}\|f(s, x(s),(H x)(s))\| d s \\
& +m\left\|\mathscr{T}\left(t-t_{k}\right) I_{k}\left(x\left(t_{k}^{-}\right)\right)\right\|,
\end{aligned}
$$

and we know that

$$
\begin{aligned}
\|f(s, x(s),(H x)(s))\| \\
\leq \mu_{3}(s)+\mu_{5}(s) \int_{0}^{s} \nu_{2}(\theta) d \theta \\
\quad+\left(\mu_{4}(s)+\mu_{5}(s) \int_{0}^{s} \nu_{3}(\theta) d \theta\right)\|x\| \\
\leq \varphi_{1}(s)+\varphi_{2}(s)\|x\| .
\end{aligned}
$$

From (50) and (51), we obtain

$$
\begin{aligned}
\|(Q x)(t)\| \leq & M_{1}\left\|x_{0}\right\|+m M_{1} \psi_{0}\|x\| \\
& +\frac{q b^{q} M_{1}}{\Gamma(1+q)} \int_{0}^{t}\left(\varphi_{1}(s)+\varphi_{2}(s)\|x\|\right) d s,
\end{aligned}
$$

where $\psi_{0}=\max \left\{\psi_{k}(t) \mid t \in I, k=1,2, \ldots, m\right\}$. Thus, for any $x \in B_{r}=\left\{x \in \mathrm{PC}[I, \mathbb{X}]:\|x\|_{\mathrm{PC}} \leq r\right\}$,

$$
\begin{aligned}
& \|(Q x)(t)\| \\
& \leq M_{1}\left\|x_{0}\right\|+\frac{q b^{q} M_{1}}{\Gamma(1+q)} \int_{0}^{b} \varphi_{1}(s) d s \\
& \quad+\left(\frac{q b^{q} M_{1}}{\Gamma(1+q)} \int_{0}^{t} \varphi_{2}(s) d s+m M \psi_{0}\right) r=\gamma_{1} .
\end{aligned}
$$

Hence, we deduce that $\|(Q x)(t)\| \leq \gamma_{1}$; that is, $Q$ maps bounded sets to bounded sets in $\operatorname{PC}[I, \mathbb{X}]$. 
Step 3. $\left(Q\left(B_{r}\right)\right.$ is equicontinuous with $B_{r}$ on $\left(t_{i}, t_{i+1}\right](i=0,1$, $2, \ldots, m))$. For any $x \in B_{r}, t^{\prime}, t^{\prime \prime} \in\left(t_{i}, t_{i+1}\right](i=0,1,2, \ldots, m)$, we obtain

$$
\begin{aligned}
\left\|(Q x)\left(t^{\prime \prime}\right)-(Q x)\left(t^{\prime}\right)\right\| \\
\leq\left\|\mathscr{T}\left(t^{\prime \prime}\right) x_{0}-\mathscr{T}\left(t^{\prime}\right) x_{0}\right\| \\
+\| \int_{0}^{t^{\prime \prime}}\left(t^{\prime \prime}-s\right)^{q-1} \mathcal{S}\left(t^{\prime \prime}-s\right) F(s) d s \\
\quad-\int_{0}^{t^{\prime}}\left(t^{\prime}-s\right)^{q-1} \mathcal{S}\left(t^{\prime}-s\right) F(s) d s \| \\
+\| \sum_{k=1}^{m} \mathscr{T}\left(t^{\prime \prime}-t_{k}\right) I_{k}\left(x\left(t_{k}^{-}\right)\right) \\
\quad-\sum_{k=1}^{m} \mathscr{T}\left(t^{\prime}-t_{k}\right) I_{k}\left(x\left(t_{k}^{-}\right)\right) \| ;
\end{aligned}
$$

after some elementary computation, we have

$$
\begin{aligned}
&\left\|(Q x)\left(t^{\prime \prime}\right)-(Q x)\left(t^{\prime}\right)\right\| \\
& \leq \| \\
&+\left\|\int_{t^{\prime}}^{t^{\prime \prime}}\left(t^{\prime \prime}\right)-\mathcal{T}\left(t^{\prime}\right)\right\|\left\|x_{0}\right\| \\
&+\| \int_{0}^{t^{\prime}}\left[\left(t^{\prime \prime}-s\right)^{q-1} \mathcal{S}\left(t^{\prime \prime}-s\right) F(s) d s \|\right. \\
&+\left\|\int_{0}^{t^{\prime}}\left(t^{\prime}-s\right)^{q-1}\left[\mathcal{S}\left(t^{\prime \prime}-s\right)-\mathcal{S}\left(t^{\prime}-s\right)\right] F(s) d s\right\| \mathcal{S}\left(t^{\prime \prime}-s\right) F(s) d s \| \\
&+m\left\|\mathscr{T}\left(t^{\prime \prime}-t^{\prime}\right)\right\|\left\|I_{k}\left(x\left(t_{k}^{-}\right)\right)\right\| .
\end{aligned}
$$

Using the fact that $\mathscr{T}(t)$ and $\mathcal{S}(t)$ are uniformly continuous, and the well-known inequality $\left|t^{\prime \sigma}-t^{\prime \prime \sigma}\right| \leq\left(t^{\prime \prime}-t^{\prime}\right)^{\sigma}$ for $\sigma \in(0,1]$ and $0<t^{\prime} \leq t^{\prime \prime}$, we can conclude that $\lim _{t^{\prime \prime} \rightarrow t^{\prime}}\left\|(Q x)\left(t^{\prime \prime}\right)-(Q x)\left(t^{\prime}\right)\right\|=0$. Thus $Q\left(B_{r}\right)$ is equicontinuous with $B_{r}$ on $\left(t_{i}, t_{i+1}\right](i=0,1,2, \ldots, m)$.

Step 4 ( $Q$ maps $B_{r}$ into a precompact set in $\mathbb{X}$ ). We define $\Pi=Q B_{r}$ and $\Pi(t)=\left\{(Q x)(t): x \in B_{r}\right\}$ for $t \in I$. Set

$$
\Pi_{h, \delta}(t)=\left\{\left(Q_{h, \delta} x\right)(t): x \in B_{r}\right\},
$$

where

$$
\begin{aligned}
\Pi_{h, \delta}(t)= & \left\{\mathscr{T}_{\delta}(t) x_{0}+\int_{0}^{t-h}(t-s)^{q-1} \mathcal{S}_{\delta}(t-s) F(s) d s\right. \\
& \left.+\sum_{k=1}^{m} \mathscr{T}_{\delta}\left(t-t_{k}\right) I_{k}\left(x\left(t_{k}^{-}\right)\right): x \in B_{r}\right\} .
\end{aligned}
$$

From Lemma 12(ii)-(iii), $\left(H_{8}\right)$, and the same method used in Theorem 3.2 of [18], we can verify that the set $\Pi(t)$ can be arbitrary approximated by the relatively compact set $\Pi_{h, \delta}(t)$. Thus, $Q\left(B_{r}\right)(t)$ is relatively compact in $\mathbb{X}$.
Step 5 (the set $E=\{x \in \mathrm{PC}[I, \mathbb{X}]: x=\lambda Q x$ for some $0<\lambda<$ $1\}$ is bounded). Let $x \in E$, and then

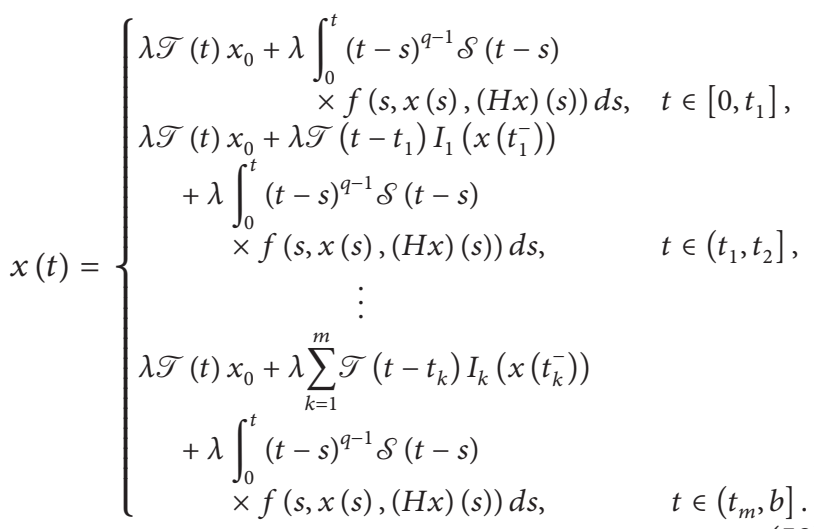

Similar to the results of (53), we know that

$$
\begin{aligned}
\|x(t)\| \leq & \lambda M_{1}\left\|x_{0}\right\|+\frac{\lambda q b^{q} M_{1}}{\Gamma(1+q)} \int_{0}^{b} \varphi_{1}(s) d s \\
& +\lambda\left(\frac{q b^{q} M_{1}}{\Gamma(1+q)} \int_{0}^{t} \varphi_{2}(s) d s+m M_{1} \psi_{0}\right)\|x(t)\| .
\end{aligned}
$$

Obviously there exists $\lambda$ sufficiently small such that $\rho=1-$ $\lambda m M_{1} \psi_{0}>0$, and then we get

$$
\begin{aligned}
\|x(t)\| \leq & \frac{\lambda M_{1}}{\rho}\left\|x_{0}\right\|+\frac{\lambda q b^{q} M_{1}}{\rho \Gamma(1+q)} \int_{0}^{b} \varphi_{1}(s) d s \\
& +\frac{\lambda q b^{q} M_{1}}{\rho \Gamma(1+q)} \int_{0}^{t} \varphi_{2}(s)\|x(s)\| d s .
\end{aligned}
$$

Let

$$
\begin{gathered}
N_{3}=\frac{\lambda M_{1}}{\rho}\left\|x_{0}\right\|+\frac{\lambda q b^{q} M_{1}}{\rho \Gamma(1+q)} \int_{0}^{b} \varphi_{1}(s) d s, \\
f(t)=\frac{\lambda q b^{q} M_{1}}{\rho \Gamma(1+q)} \int_{0}^{t} \varphi_{2}(s) d s .
\end{gathered}
$$

It is clear that $f(t)$ is nonnegative continuous function on $[0,+\infty)$, and generalized Bellman inequality implies that

$$
\|x(t)\| \leq N_{3} e^{\int_{0}^{t} f(s) d s} \leq N_{3} e^{\int_{0}^{b} f(s) d s}=C_{0},
$$

where $C_{0}$ is a constant. Obviously, the set $E$ is bounded on $\left(t_{i}, t_{i+1}\right] \quad(i=0,1,2, \ldots, m)$. Since $Q$ is continuous and compact, thanks to Schaefer's fixed point Theorem, $Q$ has a fixed point (36) which is a PC-mild solution of (4).

\section{Controllability Results}

By introducing a class of controls, we present the controllability results for fractional impulsive integrodifferential systems (6). 
$\left(H_{9}\right)$ The linear operator $W_{i}$ from $L^{2}\left[\left(t_{i-1}, t_{i}\right], U\right]$ into $\mathbb{X}$ defined by

$$
\begin{array}{r}
W_{i} u=\int_{0}^{t_{i}}\left(t_{i}-s\right)^{q-1} \mathcal{S}\left(t_{i}-s\right) B u(s) d s, \\
i=1,2, \ldots, m, m+1,
\end{array}
$$

induces an invertible operator $\widetilde{W}_{i}^{-}$defined on $L^{2}\left[\left(t_{i-1}, t_{i}\right], U\right] / \operatorname{Ker} W_{i}$, and there exists a positive constant $K>0$ such that $\left\|B \widetilde{W}_{i}^{-}\right\| \leq K$.

Theorem 15. If the hypotheses $\left(H_{1}\right)-\left(H_{3}\right),\left(H_{4}^{\prime}\right)$, and $\left(H_{9}\right)$ are satisfied, then the fractional impulsive integrodifferential system (6) is controllable on I.

Proof. Using the condition $\left(H_{9}\right)$, for an arbitrary function $x(\cdot)$, define the control

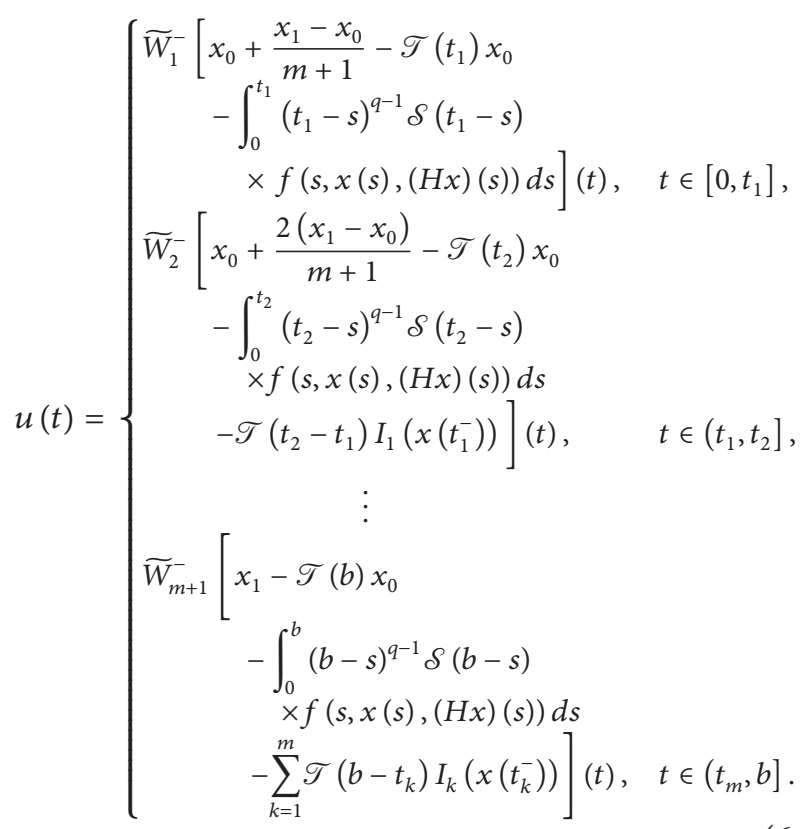

Define the operator $Q: \mathrm{PC}[I, \mathbb{X}] \rightarrow \mathrm{PC}[I, \mathbb{X}]$, where

$$
(Q x)(t)=\left\{\begin{array}{cc}
\mathscr{T}(t) x_{0}+\int_{0}^{t}(t-s)^{q-1} \mathcal{S}(t-s) & \\
\times[f(s, x(s),(H x)(s)) & \\
+B u(s)] d s, & \\
\mathscr{T}(t) x_{0}+\mathscr{T}\left(t-t_{1}\right) I_{1}\left(x\left(t_{1}^{-}\right)\right) & \\
+\int_{0}^{t}(t-s)^{q-1} \mathcal{S}(t-s) & \\
\times[f(s, x(s),(H x)(s)) & \\
+B u(s)] d s, & \\
\vdots & \\
\mathscr{T}(t) x_{0}+\sum_{k=1}^{m} \mathscr{T}\left(t-t_{k}\right) I_{k}\left(x\left(t_{k}^{-}\right)\right) & \\
+\int_{0}^{t}(t-s)^{q-1} \mathcal{S}(t-s) & \\
\times[f(s, x(s),(H x)(s)) & \\
+B u(s)] d s, & t \in\left(t_{m}, b\right] .
\end{array}\right.
$$

By Theorem 13, we know that $Q$ is well defined, and we will prove that when using the previous control, operator $Q$ has a fixed point. Clearly, this fixed point is a PC-mild solution of the control problem (6) and $x(b)=x_{1}$; that is, the control we defined steers the system (6) from initial $x_{0}$ to $x_{1}$ in the time $b$.

For any $x_{1}, x_{2} \in C\left[\left(t_{i}, t_{i+1}\right], \mathbb{X}\right](i=0,1,2, \ldots, m)$, by conditions $\left(H_{1}\right)-\left(H_{3}\right),\left(H_{4}^{\prime}\right)$, and $\left(H_{9}\right)$, we get

$$
\begin{aligned}
& \left\|B u_{1}(t)-B u_{2}(t)\right\| \\
& \leq\left(\frac{q M_{1} K}{\Gamma(1+q)} \int_{0}^{b}(b-s)^{q-1}\right. \\
& \times\left(\mu_{1}(s)+v_{1}^{0} b \mu_{2}(s)\right) d s \\
& \left.+\omega_{0} m M_{1}\right) \\
& \times\left\|x_{1}(s)-x_{2}(s)\right\|_{\mathrm{PC}} \\
& \leq \Omega_{u}\left\|x_{1}(s)-x_{2}(s)\right\|_{\mathrm{PC}}, \\
& \left\|\left(Q x_{1}\right)(t)-\left(Q x_{2}\right)(t)\right\| \\
& \leq \int_{0}^{t}(t-s)^{q-1} \| \mathcal{S}(t-s) \\
& \quad \times\left[f\left(s, x_{1}(s),\left(H x_{1}\right)(s)\right)\right. \\
& \left.\quad-f\left(s, x_{2}(s),\left(H x_{2}\right)(s)\right)\right] \| d s \\
& +\int_{0}^{t}(t-s)^{q-1} \\
& \quad \times\left\|\mathcal{S}(t-s)\left[B u_{1}(s)-B u_{2}(s)\right]\right\| d s \\
& +\sum_{k=1}^{m} \| \mathscr{T}\left(t-t_{k}\right)\left(I_{k}\left(x_{1}\left(t_{k}^{-}\right)\right)\right. \\
& \left.-I_{k}\left(x_{2}\left(t_{k}^{-}\right)\right)\right) \| .
\end{aligned}
$$

Therefore,

$$
\begin{aligned}
& \left\|\left(Q x_{1}\right)(t)-\left(Q x_{2}\right)(t)\right\| \\
& \leq\left(\frac{q M_{1}}{\Gamma(1+q)} \int_{0}^{t}(t-s)^{q-1}\left(\mu_{1}(s)+\nu_{1}^{0} b \mu_{2}(s)\right) d s\right. \\
& \left.\quad+\frac{q M_{1} \Omega_{u}}{\Gamma(1+q)} \int_{0}^{t}(t-s)^{q-1} d s+\omega_{0} m M_{1}\right) \\
& \quad \times\left\|x_{1}(s)-x_{2}(s)\right\|_{\mathrm{PC}} \\
& \leq \Omega_{m}^{\prime}(t)\left\|x_{1}(s)-x_{2}(s)\right\|_{\mathrm{PC}} .
\end{aligned}
$$

Since $0<\Omega_{m}^{\prime}(t)<1$, then $Q$ is contraction mapping. Any fixed point of $Q$ is a PC-mild solution of (6) which satisfies $x(b)=x_{1}$. Thus, the system (6) is controllable on $I$.

Theorem 16. If the hypotheses $\left(H_{5}\right)-\left(H_{7}\right),\left(H_{8}^{\prime}\right)$, and $\left(H_{9}\right)$ are satisfied, the fractional impulsive integrodifferential system (6) is controllable on $I$. 
Proof. Using the condition $\left(H_{9}\right)$, for an arbitrary function $x(\cdot)$, define the control

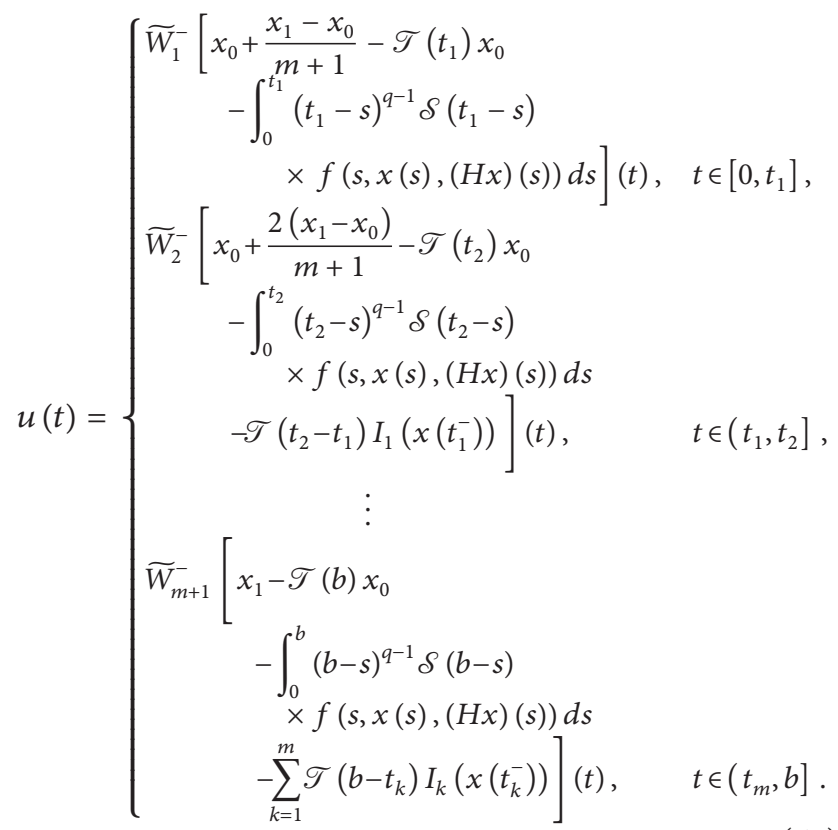

We will prove that when using the previous control, operator $Q$ defined in (65) has a fixed point.

We discuss that in five steps.

Step 1 (continuity of $Q$ on $\left.\left(t_{i}, t_{i+1}\right](i=0,1,2, \ldots, m)\right)$. Let $x_{n}, x \in \mathrm{PC}[I, \mathbb{X}]$ such that $\left\|x_{n}-x^{*}\right\|_{\mathrm{PC}} \rightarrow 0(n \rightarrow+\infty)$, and then $r=\sup _{n}\left\|x_{n}\right\|_{\mathrm{PC}}<\infty$ and $\left\|x^{*}\right\|_{\mathrm{PC}}<r$. For every $t \in\left(t_{i}, t_{i+1}\right](i=0,1,2, \ldots, m)$, we have

$$
\begin{aligned}
& \left\|Q x_{n}(t)-Q x(t)\right\| \\
& \leq \frac{q M_{1}}{\Gamma(1+q)} \int_{0}^{t}(t-s)^{q-1} \\
& \quad \times \| f\left(s, x_{n}(s),\left(H x_{n}\right)(s)\right) \\
& \quad-f(s, x(s),(H x)(s)) \| d s \\
& +\frac{q M_{1}}{\Gamma(1+q)} \int_{0}^{t}(t-s)^{q-1}\left\|B x_{n}(s)-B x(s)\right\| d s \\
& +\psi_{0} M_{1} \sum_{k=1}^{m}\left\|I_{k}\left(x_{n}\left(t_{k}^{-}\right)\right)-I_{k}\left(x\left(t_{k}^{-}\right)\right)\right\| .
\end{aligned}
$$

Since

$$
\begin{aligned}
& \left\|B x_{n}(s)-B x(s)\right\| \\
& \leq\left(\frac{q M_{1} K}{\Gamma(1+q)} \int_{0}^{b}(b-s)^{q-1}\right. \\
& \quad \times \| f\left(s, x_{n}(s),\left(H x_{n}\right)(s)\right) \\
& \quad-f(s, x(s),(H x)(s)) \| d s) \\
& +\psi_{0} M \sum_{k=1}^{m}\left\|x_{n}\left(t_{k}^{-}\right)-x\left(t_{k}^{-}\right)\right\|,
\end{aligned}
$$

by (47), (71), and the Lebesgue dominated convergence theorem, it is easy to know that

$$
\lim _{n \rightarrow \infty}\left\|\left(Q x_{n}\right)(t)-(Q x)(t)\right\|_{P C}=0
$$

Consequently, $Q$ is continuous on $\left(t_{i}, t_{i+1}\right](i=0,1,2, \ldots, m)$.

Step 2. (Q maps bounded sets into bounded sets in $\mathrm{PC}[I, \mathbb{X}]$ ). Since

$$
\begin{aligned}
& \|B u(s)\| \leq\left\|B \widetilde{W}_{i}^{-}\right\| \\
& \times\left(\left\|x_{0}\right\|+2\left\|x_{1}\right\|+M_{1}\left\|x_{0}\right\|+\frac{q M_{1}}{\Gamma(1+q)}\right. \\
& \times \int_{0}^{b}(b-s)^{q-1}\|f(s, x(s),(H x)(s))\| d s \\
& \left.+\psi_{0} M \sum_{k=1}^{m}\left\|x\left(t_{k}^{-}\right)\right\|\right) \\
& \leq\left\|B \widetilde{W}_{i}^{-}\right\| \times\left(\frac{q M_{1}}{\Gamma(1+q)} \int_{0}^{b}(b-s)^{q-1} \varphi_{1}(s) d s\left\|x_{0}\right\|\right. \\
& +2\left\|x_{1}\right\|+M_{1}\left\|x_{0}\right\|+\frac{q M_{1}\|x\|}{\Gamma(1+q)} \\
& \times \int_{0}^{b}(b-s)^{q-1} \varphi_{2}(s) d s \\
& \left.+\psi_{0} M \sum_{k=1}^{m}\left\|x\left(t_{k}^{-}\right)\right\|\right) \\
& \leq N_{1}+N_{2}\|x\|
\end{aligned}
$$

thus, from (65), we get, for any $x \in B_{r}=\{x \in \mathrm{PC}[I, \mathbb{X}]$ : $\left.\|x\|_{\mathrm{PC}} \leq r\right\}$

$$
\begin{aligned}
& \|(Q x)(t)\| \\
& \leq M_{1}\left\|x_{0}\right\|+\frac{q b^{q} M_{1}}{\Gamma(1+q)} \int_{0}^{b}\left(\varphi_{1}(s)+N_{1}\right) d s \\
& \quad+\left(\frac{q b^{q} M_{1}}{\Gamma(1+q)} \int_{0}^{b}\left(\varphi_{2}(s)+N_{2}\right) d s+m M \psi_{0}\right) r=\gamma_{2} .
\end{aligned}
$$

Hence, we deduce that $\|(Q x)(t)\| \leq \gamma_{2}$; that is, $Q$ maps bounded sets to bounded sets in $\mathrm{PC}[I, \mathbb{X}]$. Using the same method used in Theorem 14, we can verify that $Q\left(B_{r}\right)$ is equicontinuous with $B_{r}$ on $\left(t_{i}, t_{i+1}\right](i=0,1,2, \ldots, m), Q$ maps $B_{r}$ into a precompact set in $\mathbb{X}$, and $Q\left(B_{r}\right)(t)$ is relatively compact in $\mathbb{X}$. Steps 3 and 4 are omitted.

Step 5 (the set $E=\{x \in \mathrm{PC}[I, \mathbb{X}]: x=\lambda Q x$ for some $0<$ $\lambda<1\}$ is bounded). Let $x \in E$, and similar to the results (74) 
we know that

$$
\begin{aligned}
\|x(t)\| \leq & \lambda M_{1}\left\|x_{0}\right\| \\
& +\frac{\lambda q b^{q} M_{1}}{\Gamma(1+q)} \int_{0}^{t}\left(\varphi_{1}(s)+N_{1}\right) d s \\
& +\left(\frac{\lambda q b^{q} M_{1}}{\Gamma(1+q)} \int_{0}^{t}\left(\varphi_{2}(s)+N_{2}\right) d s+\lambda m M \psi_{0}\right) \\
& \times\|x(t)\| .
\end{aligned}
$$

There exists a $\lambda$ sufficiently small such that $\rho_{2}=1-\lambda m M \psi_{0}>$ 0 , and then

$$
\begin{aligned}
\|x(t)\| \leq & \frac{\lambda M_{1}}{\rho_{2}}\left\|x_{0}\right\|+\frac{\lambda q b^{q} M_{1}}{\rho_{2} \Gamma(1+q)} \int_{0}^{b}\left(\varphi_{1}(s)+N_{1}\right) d s \\
& +\frac{\lambda q b^{q} M_{1}}{\rho_{2} \Gamma(1+q)} \int_{0}^{t}\left(\varphi_{1}(s)+N_{1}\right)\|x(s)\| d s .
\end{aligned}
$$

Let

$$
\begin{gathered}
N_{4}=\frac{\lambda M_{1}}{\rho_{2}}\left\|x_{0}\right\|+\frac{\lambda q b^{q} M_{1}}{\rho_{2} \Gamma(1+q)} \int_{0}^{b}\left(\varphi_{1}(s)+N_{1}\right) d s, \\
f(s)=\frac{\lambda q b^{q} M_{1}}{\rho_{2} \Gamma(1+q)} \int_{0}^{t}\left(\varphi_{2}(s)+N_{2}\right) d s .
\end{gathered}
$$

It is clear that $f(s)$ is nonnegative continuous function on $[0,+\infty)$, and generalized Bellman inequality implies that

$$
\|x(t)\| \leq N_{4} e^{\int_{0}^{t} f(s) d s} \leq N_{4} e^{\int_{0}^{b} f(s) d s}=C_{1},
$$

where $C_{1}$ is a constant. Thus the set $E$ is bounded. Since $Q$ is continuous and compact, thanks to Schaefer's fixed point Theorem, $Q$ has a fixed point (36), and this fixed point is a PC-mild solution of (6) which satisfies $x(b)=x_{1}$. Hence, the system (6) is controllable on $I$.

\section{An Example}

Consider the following nonlinear partial integrodifferential equation of the form

$$
\begin{aligned}
\frac{\partial^{2 / 3}}{\partial t^{2 / 3} z(t, y)=} & \int_{0}^{1}(y-s) z(s, y) d s \\
& +f(t, z(t, y), H z(t, y)) \\
& +\mu(t, y), \quad t \in J=[0,1], \\
z(t, 0) & =z(t, 1)=0, \\
z(0, y) & =0, \quad 0<y<1, \\
\left.\Delta z\right|_{t=1 / 2} & =I_{1}\left(z\left(\frac{1}{2}^{-}, y\right)\right),
\end{aligned}
$$

where $0<q<1, \mu: J \times(0,1) \rightarrow(0,1)$ is continuous. Let us take $\mathbb{X}=C([0,1])$. Consider the operator $A: D(A) \subset \mathbb{X} \rightarrow$ $\mathbb{X}$ defined by

$$
(A w)(t)=\int_{0}^{1}(y-s) w(s) d s
$$

It is easy to get

$$
\|A w\|=\|w\| \int_{0}^{1}|y-s| d s=\left(\frac{1}{2}-y(1-y)\right)\|w\| \leq \frac{1}{2}\|w\| ;
$$

clearly $A$ is the infinitesimal generator of a uniformly continuous semigroup $(T(t))_{t \geq 0}$ on $\mathbb{X}$. Put $x(t)=z(t, \cdot)$ and $u(t)=\mu(t, \cdot)$, and take

$$
\begin{aligned}
& f(t, x, H x)= e^{t}+a(t)\left(\frac{\|x\|}{1+\|x\|}\right) \\
&+\int_{0}^{t} k(t, s)\left(\frac{\|x\|}{1+\|x\|}\right) d s, \\
& I_{1}(x)=\|x\|,
\end{aligned}
$$

where $a(t) \in C[0,1], k(t, s) \in C([0,1] \times[0,1])$. Then clearly, $f:[0,1] \times \mathbb{R} \times \mathbb{R} \rightarrow \mathbb{R}$ and $I_{1}: \mathbb{R} \rightarrow \mathbb{R}$ are continuous functions. $f, I_{1}$, and $h$ satisfy $\left(H_{5}\right)-\left(H_{8}^{\prime}\right)$, respectively. Equations (79) are an abstract formulation of (6). For $y \in(0,1)$, we define

$$
\begin{gathered}
W_{1} u=\int_{0}^{1 / 2}\left(\frac{1}{2}-s\right)^{-1 / 3} \mathcal{S}\left(\frac{1}{2}-s\right) B u(s) d s, \\
W_{2} u=\int_{0}^{1}(1-s)^{-1 / 3} \mathcal{S}(1-s) B u(s) d s,
\end{gathered}
$$

where

$$
\begin{gathered}
\mathscr{T}(t) w(s)=\int_{0}^{\infty} \xi_{2 / 3}(\theta) w\left(t^{2 / 3} \theta+s\right) d \theta, \\
\mathcal{S}(t) w(s)=\frac{2}{3} \int_{0}^{\infty} \theta \xi_{2 / 3}(\theta) w\left(t^{2 / 3} \theta+s\right) d \theta,
\end{gathered}
$$

and for $\theta \in(0, \infty)$,

$$
\begin{gathered}
\xi_{2 / 3}(\theta)=\frac{3}{2} \theta^{-5 / 2} \omega_{2 / 3}\left(\theta^{-3 / 2}\right), \\
\omega_{2 / 3}(\theta)=\frac{1}{\pi} \sum_{n=1}^{\infty}(-1)^{n-1} \theta^{-((2 n+3) / 3)} \frac{\Gamma((2 n+3) / 3)}{n !} \sin \left(\frac{2 n \pi}{3}\right) .
\end{gathered}
$$

Assume that the linear operator $W_{i}$ from $L^{2}\left[\left(t_{i-1}, t_{i}\right], U\right](i=$ $1,2)$ into $\mathbb{X}$ induces an invertible operator $\widetilde{W}_{i}^{-}$defined on $L^{2}\left[\left(t_{i-1}, t_{i}\right], U\right] / \operatorname{Ker} W_{i}$ and there exists a positive constant $K>0$ such that $\left\|B \widetilde{W}_{i}^{-}\right\| \leq K$. Moreover, $\left(H_{9}\right)$ is satisfied. All conditions of Theorem 16 are now fulfilled, so we deduce that (79) is controllable on $I$. On the other hand, we have

$$
\begin{gathered}
\|f(t, x, H x)-f(t, y, H y)\| \\
\leq a(t)\|x-y\|+\int_{0}^{t} k(t, s)\|x-y\| d s, \\
k(t, s)\|x\|-k(t, s)\|y\| \leq k_{0}(\|x\|-\|y\|), \\
k_{0}=\max \{k(t, s) \mid(t, s) \in I \times I\}, \\
\left\|I_{1}(x)-I_{1}(y)\right\| \leq\|x-y\| .
\end{gathered}
$$


Further, other conditions $\left(H_{1}\right)-\left(H_{3}\right)$ are satisfied and it is possible to choose $a(t), k(t, s)$ in such a way that condition $\left(H_{4}^{\prime}\right)$ is satisfied. Hence, by Theorem 15 , the system (79) is controllable on $I$.

\section{References}

[1] I. Podlubny, Fractional Differential Equations, vol. 198 of Mathematics in Science and Engineering, Academic Press, San Diego, Calif, USA, 1999.

[2] A. A. Kilbas, H. M. Srivastava, and J. J. Trujillo, Theory and Applications of Fractional Differential Equations, vol. 204 of North-Holland Mathematics Studies, Elsevier Science B.V., Amsterdam, The Netherlands, 2006.

[3] K. S. Miller and B. Ross, An Introduction to the Fractional Calculus and Fractional Differential Equations, John Wiley \& Sons, New York, NY, USA, 1993.

[4] V. Lakshmikantham, S. Leela, and J. Vasundhara Devi, Theory of Fractional Dynamic Systems, Cambridge Scientific Publishers, Cambridge, UK, 2009.

[5] Z.-W. Lv, J. Liang, and T.-J. Xiao, "Solutions to the Cauchy problem for differential equations in Banach spaces with fractional order," Computers \& Mathematics with Applications, vol. 62, no. 3, pp. 1303-1311, 2011.

[6] C. Kou, H. Zhou, and Y. Yan, "Existence of solutions of initial value problems for nonlinear fractional differential equations on the half-axis," Nonlinear Analysis: Theory, Methods \& Applications, vol. 74, no. 17, pp. 5975-5986, 2011.

[7] X. Su, "Solutions to boundary value problem of fractional order on unbounded domains in a Banach space," Nonlinear Analysis: Theory, Methods \& Applications, vol. 74, no. 8, pp. 2844-2852, 2011.

[8] N. Kosmatov, "Integral equations and initial value problems for nonlinear differential equations of fractional order," Nonlinear Analysis: Theory, Methods \& Applications, vol. 70, no. 7, pp. 25212529, 2009.

[9] S. Liang and J. Zhang, "Positive solutions for boundary value problems of nonlinear fractional differential equation," Nonlinear Analysis: Theory, Methods \& Applications, vol. 71, no. 11, pp. 5545-5550, 2009.

[10] Y. Wang, L. Liu, and Y. Wu, "Positive solutions for a nonlocal fractional differential equation," Nonlinear Analysis: Theory, Methods \& Applications, vol. 74, no. 11, pp. 3599-3605, 2011.

[11] D. Baĭnov and P. Simeonov, Impulsive Differential Equations: Periodic Solutions and Applications, vol. 66 of Pitman Monographs and Surveys in Pure and Applied Mathematics, Longman Scientific \& Technical, Harlow, UK, 1993.

[12] V. Lakshmikantham, D. D. Bănov, and P. S. Simeonov, Theory of Impulsive Differential Equations, vol. 6 of Series in Modern Applied Mathematics, World Scientific Publishing, Teaneck, NJ, USA, 1989.

[13] M. Benchohra, J. Henderson, and S. Ntouyas, Impulsive Differential Equations and Inclusions, vol. 2 of Contemporary Mathematics and Its Applications, Hindawi Publishing Corporation, New York, NY, USA, 2006.

[14] K. Balachandran and J. P. Dauer, "Controllability of nonlinear systems in Banach spaces: a survey," Journal of Optimization Theory and Applications, vol. 115, no. 1, pp. 7-28, 2002.

[15] Z. Tai and X. Wang, "Controllability of fractional-order impulsive neutral functional infinite delay integrodifferential systems in Banach spaces," Applied Mathematics Letters, vol. 22, no. 11, pp. 1760-1765, 2009.

[16] Z. Yan, "Controllability of fractional-order partial neutral functional integrodifferential inclusions with infinite delay," Journal of the Franklin Institute, vol. 348, no. 8, pp. 2156-2173, 2011.

[17] K. Balachandran and J. Y. Park, "Controllability of fractional integrodifferential systems in Banach spaces," Nonlinear Analysis: Hybrid Systems, vol. 3, no. 4, pp. 363-367, 2009.

[18] J. Wang and Y. Zhou, "Complete controllability of fractional evolution systems," Communications in Nonlinear Science and Numerical Simulation, vol. 17, no. 11, pp. 4346-4355, 2012.

[19] G. M. Mophou, "Existence and uniqueness of mild solutions to impulsive fractional differential equations," Nonlinear Analysis: Theory, Methods \& Applications, vol. 72, no. 3-4, pp. 1604-1615, 2010.

[20] E. Hernández, D. O’Regan, and K. Balachandran, “On recent developments in the theory of abstract differential equations with fractional derivatives," Nonlinear Analysis: Theory, Methods \& Applications, vol. 73, no. 10, pp. 3462-3471, 2010.

[21] O. K. Jaradat, A. Al-Omari, and S. Momani, "Existence of the mild solution for fractional semilinear initial value problems," Nonlinear Analysis: Theory, Methods \& Applications, vol. 69, no. 9, pp. 3153-3159, 2008.

[22] J. Wang, M. Fečkan, and Y. Zhou, "On the new concept of solutions and existence results for impulsive fractional evolution equations," Dynamics of Partial Differential Equations, vol. 8, no. 4, pp. 345-361, 2011.

[23] J. Wang and Y. Zhou, "A class of fractional evolution equations and optimal controls," Nonlinear Analysis: Real World Applications, vol. 12, no. 1, pp. 262-272, 2011.

[24] Y. Zhou and F. Jiao, "Existence of mild solutions for fractional neutral evolution equations," Computers \& Mathematics with Applications, vol. 59, no. 3, pp. 1063-1077, 2010.

[25] A. Pazy, Semigroups of Linear Operators and Applications to Partial Differential Equations, vol. 44 of Applied Mathematical Sciences, Springer, New York, NY, USA, 1983. 


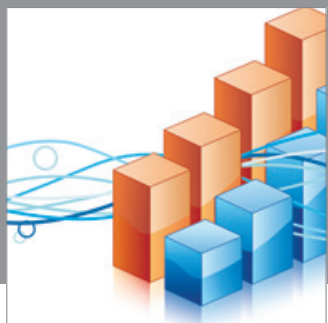

Advances in

Operations Research

mansans

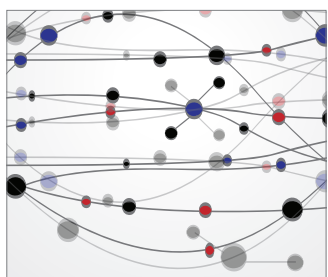

The Scientific World Journal
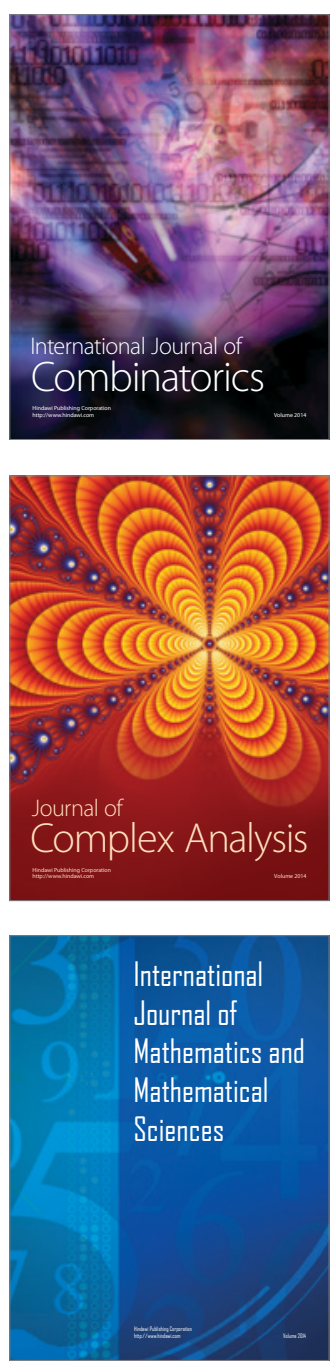
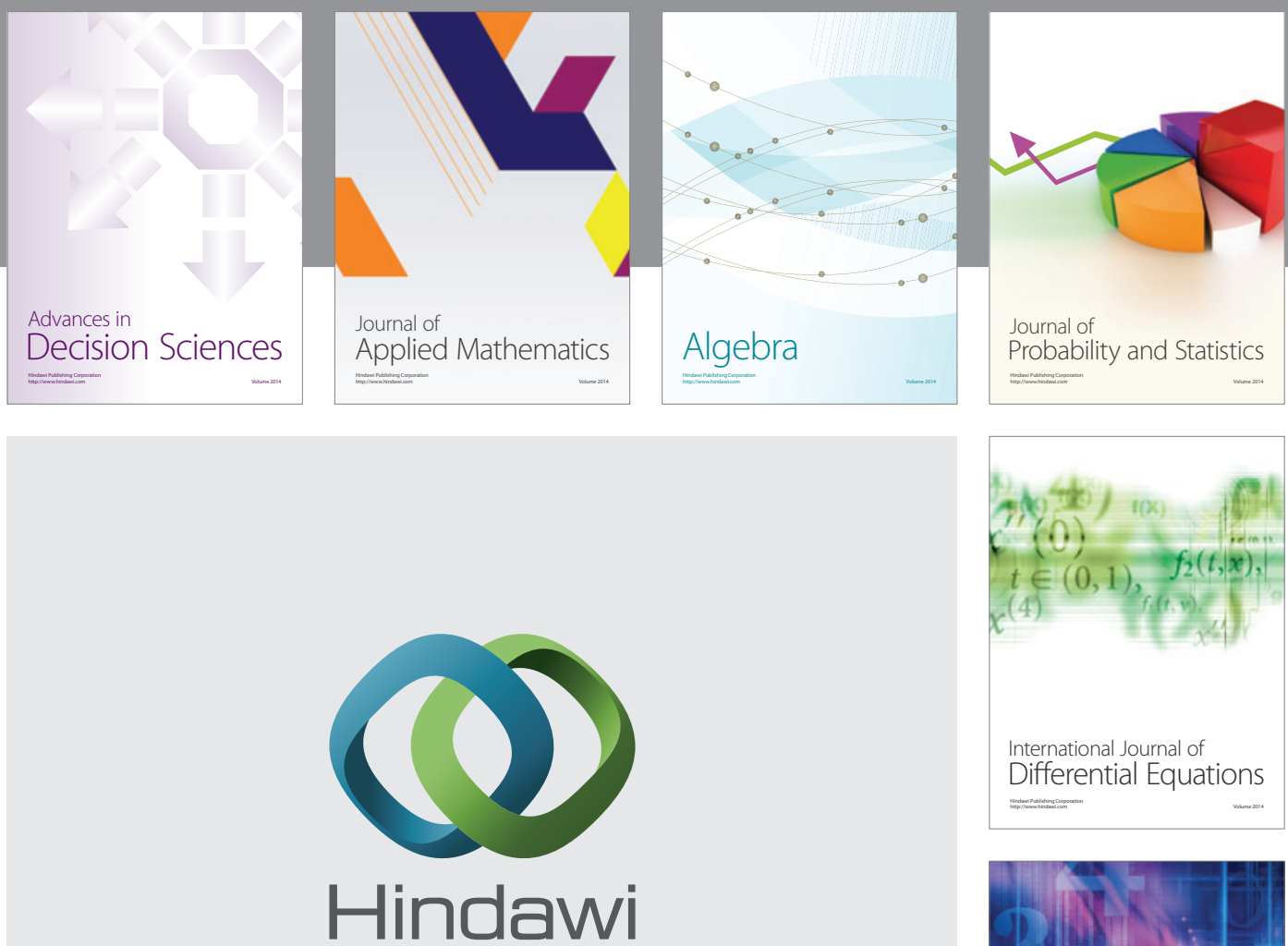

Submit your manuscripts at http://www.hindawi.com
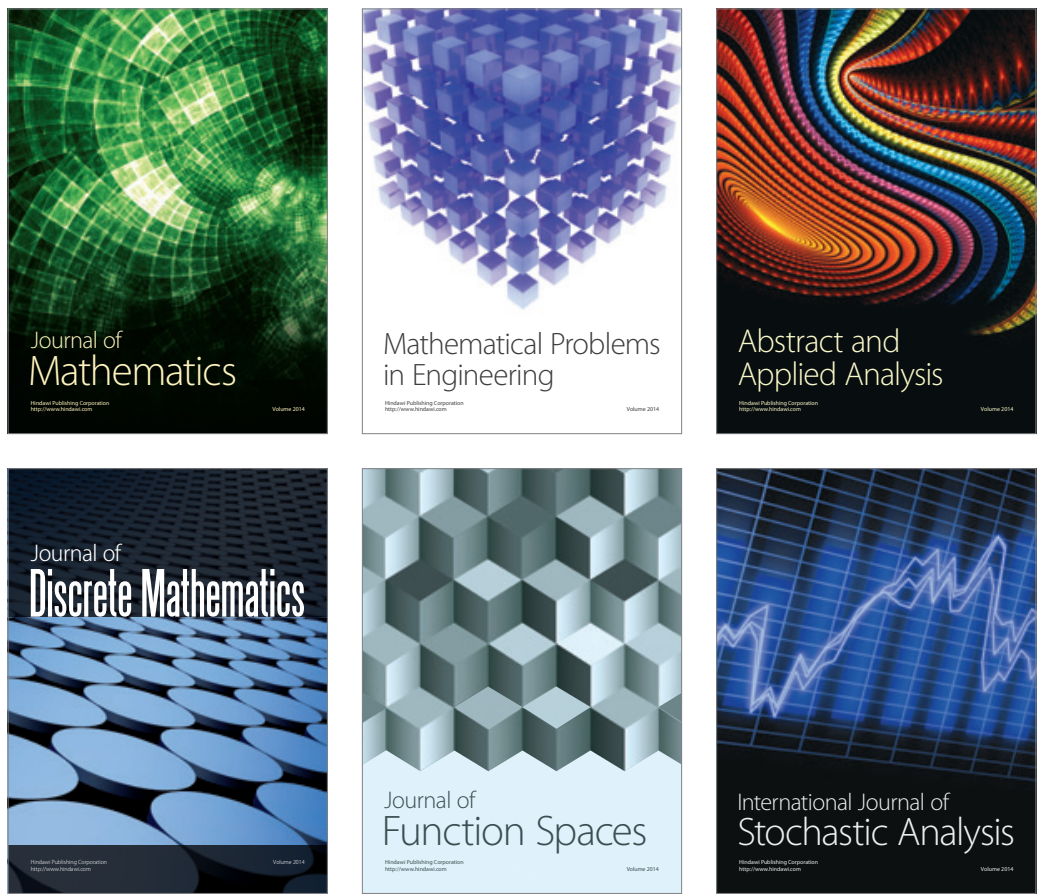

Journal of

Function Spaces

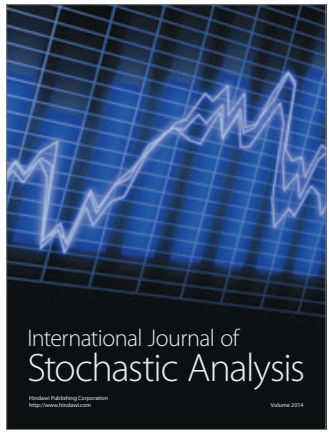

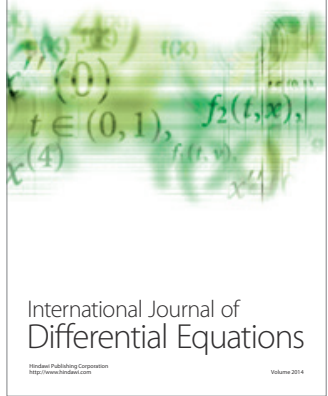
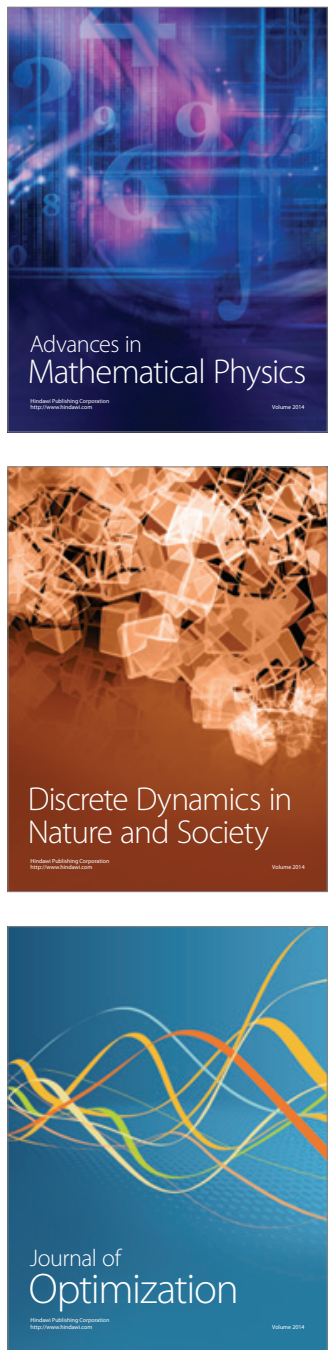